\title{
James Young Simpson Symposium: Maternal Medicine
}

\author{
SJ Stock \\ Clinical Lecturer and Subspecialty Trainee, University of Edinburgh Centre for Reproductive Health, Queen's Medical Research Institute, \\ Edinburgh, UK
}

\begin{abstract}
The James Young Simpson Bicentenary Celebrations RCPE Symposium on Maternal Medicine, in association with the Royal College of Obsterics and Gynaecology, was held in Edinburgh on 2 June $20 \mathrm{II}$.
\end{abstract}

DECLARATION OF INTERESTS No conflict of interests declared.

\author{
Correspondence to SJ Stock, \\ Room S7 I 29, Simpson Centre \\ for Reproductive Health, \\ Royal Infirmary of Edinburgh, \\ Little France, Edinburgh, \\ EHI6 4SA, UK
}

tel. +44 (0) 13। 242269 I

e-mail sarah.stock@ed.ac.uk

\section{INTRODUCTION}

Doctors and midwives are increasingly faced with pregnant women with complex medical conditions. The average age of first pregnancy is rising and the prevalence of chronic conditions such as diabetes, obesity and cardiac disease in the childbearing population is going up. The pattern of pregnancy complications is also changing, with sepsis emerging as the most common cause of direct maternal death. This multidisciplinary meeting focused on advances in the care of pregnant women with medical complications and the ongoing challenges to improving long-term outcomes for these women.

\section{SESSION I}

Peripartum maternal sepsis

Professor Shiranee Sriskandan (Professor of Infectious Diseases, Imperial College, London) highlighted that since 1985 there has been close to a three-fold rise in the number of maternal deaths related to peripartum sepsis, from 0.4 to 1.13 per 100,000 maternities.' This mainly relates to Group A streptococcal (GAS) sepsis, the risk of which is more than 120 times higher in the immediate postpartum period. There is a need to educate all healthcare providers to recognise the relevance of recent pregnancy in a woman presenting with signs of infection. Prompt appropriate antibiotic treatment with GAS cover is essential. Clindamycin is an agent of choice due to its superior tissue penetration and capacity to inhibit GAS toxin synthesis.

\section{HIV in pregnancy}

Dr Hermione Lyall (Consultant Paediatrician, Imperial College Healthcare NHS Trust, London) described the success of interventions to decrease mother-to-child transmission of HIV, with transmission rates in the UK now below I\%. The use of highly active anti-retroviral therapy (HAART) from conception can reduce transmission rates to $0.1 \%$. Concerns about possible teratogenicity seem unfounded, with the risk of severe congenital anomalies associated with first trimester HAART similar to that of second/third trimester. ${ }^{2}$ Nevertheless, HAART is associated with increased risk of premature delivery, with rates of preterm birth before 37 weeks at 1.5 times higher in women on HAART than on mono or dual therapy. ${ }^{3}$ Babies born preterm are at higher risk of HIV, partly due to difficulties in delivering effective post-exposure prophylaxis intravenously. An important learning point for obstetricians is to 'preload' mothers with threatened preterm labour with agents such as nevirapine, that can help protect the fetus for more than a week after birth.

New data from the UK suggest that if women are receiving effective HIV treatment, a more relaxed approach could be taken if there is slow progress in labour. In women with undetectable viral loads, motherto-child HIV transmission rates were low even in cases where rupture of membranes exceeded six hours. However, evidence that breastfeeding increases transmission even when HAART and post-exposure prophylaxis are used, means that in developed countries advice to bottle feed should remain in place.

\section{Critical care of the acutely ill pregnant woman}

Dr Felicity Plaat (Consultant Anaesthetist, Hammersmith Hospital, London) argued that high-dependency care does not need to be confined to a specific area in the hospital and critical care should start on the obstetric unit as soon as it is indicated. The lack of a standard definition for critical care in obstetrics remains a barrier to securing funding and service planning. However, it can decrease inappropriate intensive care admissions and ensures involvement of specialists with knowledge of obstetric conditions. It also helps continuity of care and keeps mother and baby together. Good communication, a team approach and the involvement of senior medical staff are crucial. Simulation-based team training is associated with improvement in outcomes. ${ }^{4}$ 


\section{SESSION 2}

\section{Preventing maternal deaths in pregnancy due to heart disease}

Dr Lorna Swan (Consultant Cardiologist, Royal Brompton Hospital, London) provided a cardiologist's perspective on the most recent confidential enquiry on maternal mortality.' Advanced therapies have led to a reduction in mortality associated with congenital heart disease ${ }^{5}$ but cases of acquired cardiac diseases are increasing. Educating both the public and healthcare professionals that pregnancy is a risk factor for cardiovascular disease is key. Ischaemic heart disease, aortic dissection and myocardial disease should be considered in the differential diagnosis of pregnant women presenting with chest pain. Appropriate investigations must not be avoided because of pregnancy, but interpretation may be complicated by pregnancy so experienced senior staff should be involved. Urgent transfer for primary coronary investigation is indicated if there is suspicion of myocardial infarction. Sudden arrhythmic death syndrome (SADS) is recognised as a cause of death in pregnancy that is difficult to predict or prevent. In these cases, specialist postmortem with retention of samples for genetic investigations is important because it may help diagnosis in other family members.

\section{Hypertension in pregnancy}

Professor Stephen Robson, Professor of Fetal Medicine, Newcastle University delivered the Sydney Watson Smith lecture, which focused on pre-eclampsia. He introduced the concept of a 'disease of placental origin' which may affect the fetus, as well as multiple organ systems in the mother. The phenotype depends on both the placental stimulus and the maternal response to it, explaining pre-eclampsia's variable onset and severity. Clinical definitions now reflect this. Only $50 \%$ of women with pre-eclampsia present with the classical signs of raised blood pressure and proteinuria.

Measures to predict and prevent pre-eclampsia must be instituted in early pregnancy to be effective. If commenced before 16 weeks, aspirin is associated with a 0.47 relative risk $(0.34-0.65)$ of pre-eclampsia. ${ }^{6}$ Combinations of first trimester markers for prediction of pre-eclampsia show promise and an important new trial of first trimester pregnancy associated plasma protein A (PAPP-a) screening and aspirin treatment is to be launched.

The aims of management of pre-eclampsia include reduction of maternal morbidity and timely delivery. Magnesium sulphate is the treatment of choice for eclampsia, and data suggest prophylactic use can decrease the risk of first fit by $60 \%$. It is also neuroprotective for the fetus, so its use as a preventative agent, especially in preterm pre-eclampsia is advocated. The recent hypertension and pre-eclapmsia intervention trial at term (HYPITAT) study has suggested that maternal adverse outcomes are reduced if women with preeclampsia or gestational hypertension are delivered at 36 weeks, with no difference in caesarean section rate or neonatal unit admission. ${ }^{7}$

A future challenge is how to reduce the long-term risks in women who 'failed the pregnancy test' and developed pre-eclampsia. Risks of cardiovascular disease are 2-3 fold higher in women who have pre-eclampsia. ${ }^{8,9}$ Postnatal follow-up with provision of lifestyle advice and pharmacological intervention in women with additional risk factors may be one strategy to improving lifelong health for mothers.

\section{SESSION 3: INTERACTIVE WORKSHOPS}

The third session consisted of interactive workshops to illustrate multidisciplinary management of maternal medical presentations in pregnancy. Dr Roch Cantwell (Consultant Perinatal Psychiatrist, Southern General Hospital, Glasgow) led a workshop on psychiatric disease in pregnancy. Dr Liam Plant (Consultant Renal Physician, Cork University Hospital, Ireland) provided a workshop on renal disease in pregnancy. Dr Richard Davenport (Consultant Neurologist, Western General Hospital, Edinburgh) gave a workshop on headache in pregnancy.

\section{SESSION 4: CASE PRESENTATIONS}

Professor Catherine Nelson-Piercy (Consultant Obstetric Physician, Guy's and St. Thomas' NHS Foundation Trust, London) chaired a panel discussion (Dr Richard Davenport, Dr Liam Plant, Professor Shiranee Sriskandan) of four case presentations.

Dr Nithiya Palaniappan (ST6 Obstetrics and Gynaecology, Royal Infirmary Edinburgh) presented a case of Type I diabetes in pregnancy complicated by HINI pneumonia. The controversies regarding timing of delivery in the compromised pregnant woman were discussed. A retrospective series has suggested that in severe HINI pneumonitis, delivery was associated with improvements in recovery.

Dr Leena Thomas (ST5 Obstetrics and Gynaecology, Aberdeen Royal Infirmary) presented a case of sickle cell disease in pregnancy. The case illustrated diagnostic uncertainty in women with sickle cell disease presenting with chest pain, where the differential diagnosis includes infection, pulmonary embolus and acute chest syndrome. A reminder was given that in the case of an abnormal chest $X$-ray, computed tomography pulmonary angiography is the radiological investigation of choice for pulmonary embolus.

Dr Gemma Sheridan (ST5 Wirral University Teaching Hospital, NHS Foundation Trust) presented a case of group A streptococcal septicaemia. The case 
demonstrated how the use of early warning scores (EWS) charts can help identify deteriorating patients, with increasing respiratory rate a particularly worrying feature. It also illustrated the importance of lactate measurement and C-reactive protein in assessment of the patient with sepsis.

Dr Faiza Wali Ullah (ST4/LAT Obstetrics and Gynaecology, Stirling Royal Infirmary) presented a case of hereditary haemorrhagic telangiectasia associated with pregnancy. The panel discussed the rationale for screening for intracranial arteriovenous malformations (AVM). They felt that not enough is known about the natural history of intracranial AVM to recommend screening, and cerebral magnetic resonance imaging (MRI) should only be performed if symptoms are present. However an MRI of the spine may be useful as the identification of AVM would preclude regional anaesthesia.

\section{REFERENCES}

I Cantwell R, Clutton-Brock T, Cooper G. Centre for Maternal and Child Enquiries (CMACE). Saving mothers' lives: reviewing maternal deaths to make motherhood safer: 2006-08. The eighth report on confidential enquiries into maternal deaths in the United Kingdom. BJOG 201 I; I 18:1-203.

2 Antiretroviral Pregnancy Registry Steering Committee. Antiretroviral Pregnancy Registry International. Interim Report for I January 1989 through 3I July 2009 [Internet]. Wilmington: The Antiretroviral Pregnancy Register; 2009 [cited 201I Sept 12]. Available from: http://www.apregistry.com/

3 Townsend CL, Cortina-Borja M, Peckham CS et al. Low rates of mother-to-child transmission of HIV following effective pregnancy interventions in the United Kingdom and Ireland, 2000-2006. AIDS 2008;22:973-81 http://dx.doi.org/10.1097/QAD.0b0 I3e3282f9b67a

4 Siassakos D, Hasafa Z, Sibanda T et al. Retrospective cohort study of diagnosis-delivery interval with umbilical cord prolapse: the effect of team training. BJOG 2009; I16:1089-96. http://dx.doi. org/I0.IIII/j. I 47I-0528.2009.02I79.x

\section{TAKE-HOME MESSAGE}

The symposium provided strategies for managing medical conditions that can be encountered when caring for pregnant women. It reinforced how crucial it is for all healthcare providers to recognise the significance of ongoing and recent pregnancy as risk factors for medical complications. Cases highlighted the importance of multidisciplinary care and effective communication between specialties for successful management of complex cases and reduction in morbidity.

5 Bédard E, Shore DF, Gatzoulis MA. Adult congenital heart disease: a 2008 overview. $\mathrm{Br}$ Med Bull 2008; 85:I5I-80. http://dx.doi. org/10.1093/bmb/ldn005

6 Bujold E, Roberge S, LacasseY et al. Prevention of pre-eclampsia and intrauterine growth restriction with aspirin started in early pregnancy: a meta-analysis. Obstet Gynecol 2010; I 16:402-14. http:// dx.doi.org/I0.1097/AOG.0b0I3e3I8Ie9322a

7 Koopmans CM, Bijlenga D, Groen $\mathrm{H}$ et al. Induction of labour versus expectant monitoring for gestational hypertension or mild pre-eclampsia after 36 weeks' gestation (HYPITAT): a multicentre, open-label randomised controlled trial. Lancet 2009; 374:979-88. http://dx.doi.org/I0.1016/S0I40-6736(09)60736-4

8 Smith GC, Pell JP, Walsh D. Pregnancy complications and maternal risk of ischaemic heart disease: a retrospective cohort study of 129,290 births. Lancet 200 |; 357:2002-6. http://dx.doi.org/I0.1016/ S0I40-6736(00)05 I I 2-6

9 Irgens HU, Reisaeter L, Irgens LM et al. Long term mortality of mothers and fathers after pre-eclampsia: population based cohort study. BMJ 200I; 323:I213-17. http://dx.doi.org/I0.I I36/bmj.323.7323.I2I3 